\title{
Omitting breakfast and lunch after injection of different long-acting insulin preparations at bedtime: a prospective study in patients with type 2 diabetes
}

\author{
P. Wiesli • P. Krayenbühl • H. Uthoff • B. Seifert • \\ C. Schmid
}

Received: 8 June 2009 /Accepted: 11 June 2009 /Published online: 30 June 2009

(C) Springer-Verlag 2009

\begin{abstract}
Aims/hypothesis The aim of this prospective trial was to compare the effect of different long-acting insulin preparations injected at bedtime on glucose concentrations in patients with type 2 diabetes omitting breakfast and lunch the next day.

Methods Twenty patients (ten women) with type 2 diabetes who were on an intensified insulin therapy participated. Mean $( \pm \mathrm{SD})$ age was $63 \pm 10$ years, diabetes duration $18 \pm$ 9 years, BMI $32.5 \pm 5 \mathrm{~kg} / \mathrm{m}^{2}$, and $\mathrm{HbA}_{1 \mathrm{c}} 7.3 \pm 0.7 \%$. Patients received neutral protamine Hagedorn (NPH) insulin, insulin detemir or insulin glargine for at least 2 months; doses were adjusted to achieve morning blood glucose levels of $<7 \mathrm{mmol} / \mathrm{l}$. At the end of the respective treatment period, the long-acting insulin was injected at bedtime (at 22:45 hours) as usual but patients refrained from breakfast and
\end{abstract}

P. Wiesli $(\bowtie) \cdot$ H. Uthoff

Medizinische Klinik, Endokrinologie und Diabetologie,

Kantonsspital Frauenfeld,

8500 Frauenfeld, Switzerland

e-mail: peter.wiesli@stgag.ch

P. Krayenbühl

Department of Internal Medicine, Medical Policlinic,

University Hospital Zurich,

Zurich, Switzerland

B. Seifert

Biostatistics Unit, Institute of Social and Preventive Medicine,

University of Zurich,

Zurich, Switzerland

C. Schmid

Department of Internal Medicine,

Division of Endocrinology and Diabetes,

University Hospital Zurich,

Zurich, Switzerland lunch the next day; glucose was measured by a continuous glucose monitoring system (CGMS).

Results Comparable glucose target ranges were reached at midnight $(5.8$ to $6.1 \mathrm{mmol} / \mathrm{l})$ and at $07: 00$ hours $(6.7$ to $6.9 \mathrm{mmol} / \mathrm{l})$ with all three insulin preparations, using mean doses of $29 \pm 10 \mathrm{U}$ (NPH insulin), $33 \pm 13 \mathrm{U}$ (insulin detemir), and $32 \pm 12 \mathrm{U}$ (insulin glargine). Glucose levels between midnight and 07:00 hours were not significantly different for the three insulin preparations. Symptomatic hypoglycaemia did not occur from 08:00 to 16:00 hours; glucose concentrations during this time were slightly lower with NPH insulin than with insulin detemir $(p=0.012)$ and insulin glargine $(p=0.049)$.

Conclusions/interpretation Following bedtime injection of $\mathrm{NPH}$ insulin or of the analogues insulin detemir or insulin glargine, fasting glucose $<7 \mathrm{mmol} / \mathrm{l}$ was achieved in the morning, without subsequent hypoglycaemia when participants continued to fast during the day.

Keywords Insulin detemir - Insulin glargine - NPH insulin . Type 2 diabetes
Abbreviations
CGMS Continuous glucose monitoring system
CSII Continuous subcutaneous insulin infusion
NPH Neutral protamine Hagedorn

\section{Introduction}

In patients with type 1 diabetes or healthy individuals, the action of insulin detemir and insulin glargine has been found to be more stable and more prolonged than that of neutral protamine Hagedorn (NPH) insulin [1-3], but such 
findings cannot necessarily be extrapolated to patients with type 2 diabetes. Findings from glucose clamp studies of long-acting insulin analogues are difficult to interpret and are of questionable relevance for clinical practice in patients with type 2 diabetes [4]. Despite scant comparative data showing clinical superiority over NPH insulin, long-acting insulin analogues are increasingly used in type 2 diabetes [5].

Insulin requirements follow circadian rhythms, with an increase in the early morning hours [6-8]. For the patient with type 2 diabetes, an insulin preparation injected at bedtime needs to have a duration of action until the next morning but not beyond $12 \mathrm{~h}$, provided that meal-related insulin requirements are covered by oral hypoglycaemic agents or short-acting insulin. A prolonged duration of action of insulin preparations injected at bedtime could induce hypoglycaemia when patients omit breakfast next day.

The goal of this study was to compare the effect of different long-acting insulin preparations injected at bedtime on glucose concentrations overnight and the next day when patients with type 2 diabetes remained fasting.

\section{Methods}

Patients This single-centre study recruited patients at University Hospital Zurich. Twenty patients with type 2 diabetes who were on intensified insulin therapy were included in the study. The baseline characteristics of the patients are shown in Table 1. Eleven patients had microalbuminuria but all had an estimated glomerular filtration rate (using the Cockroft-Gault formula) of $>50 \mathrm{ml} / \mathrm{min}$ [9]. The Ethics Committee of the University Hospital of Zurich approved the study and all patients gave written informed consent.

Study protocol In this prospective non-blinded study, each patient was treated for at least 2 months with NPH insulin, insulin detemir (NN304) [B29Lys( $\varepsilon$-tetradecanoyl), desB30 human insulin] and insulin glargine (A21Gly,B31Arg, B32Arg human insulin) injected at bedtime. The longacting insulin used at the time of recruitment was tested first, and the dose was initially reduced by $20 \%$. After completion of the fasting test, the next long-acting insulin was given in the following order: NPH insulin, insulin detemir, insulin glargine. The same starting dose was administered with all three preparations.

Therapy results were discussed with the patients by telephone at weekly intervals, and the dose was adjusted according to reported glucose concentrations. The goal of therapy was to achieve blood glucose levels of 5 to $8 \mathrm{mmol} / \mathrm{l}$ at bedtime and fasting blood glucose values of $<7 \mathrm{mmol} / \mathrm{l}$. When
Table 1 Baseline characteristics of 20 patients with type 2 diabetes mellitus

\begin{tabular}{ll}
\hline Characteristic & Value \\
\hline Sex (number of males/females) & $10 / 10$ \\
Age (years) & $63 \pm 10$ \\
BMI $\left(\mathrm{kg} / \mathrm{m}^{2}\right)$ & $32.5 \pm 5$ \\
Weight $(\mathrm{kg})$ & $93 \pm 16$ \\
Diabetes duration (years) & $18 \pm 9$ \\
Duration of insulin therapy (years) & $9 \pm 8$ \\
Daily insulin dose (U/day) & $66 \pm 18$ \\
HbA $1 \mathrm{c}(\%)$ & $7.3 \pm 0.7^{\mathrm{a}}$ \\
Creatinine ( $\mu$ mol/l) & $91 \pm 12^{\mathrm{b}}$ \\
Retinopathy & $10(50)$ \\
Nephropathy & $11(55)$ \\
Neuropathy & $16(80)$ \\
Macroangiopathy & $7(35)$ \\
\hline
\end{tabular}

Data are presented as mean \pm SD or $n(\%)$

${ }^{\text {a Normal range } 4-6 \%}$

${ }^{\mathrm{b}}$ Normal range in women $60-90 \mu \mathrm{mol} / \mathrm{l}$; in men $70-105 \mu \mathrm{mol} / 1$

the fasting blood glucose level was $<7 \mathrm{mmol} / \mathrm{l}$ on at least 3 days per week and if the patient had been treated for a minimum of 2 months with the long-acting insulin, a fasting test was performed.

A Medtronic MiniMed Continuous Glucose Monitoring System (CGMS; Medtronic, Northridge, CA, USA) was attached to the patients in the evening before the fasting test. The long-acting insulin was injected at the usual dose at bedtime. No breakfast or lunch was consumed on the test day. The patients were advised to drink liquids (water) and to continue with their normal daily activities, and were instructed to discontinue the test if symptoms of hypoglycaemia and a capillary blood glucose value of $<4 \mathrm{mmol} / \mathrm{l}$ occurred. From 08:00 to 16:00 hours the patients determined their capillary blood glucose value every $2 \mathrm{~h}$ (Accu-Chek; Roche Diagnostics, Mannheim, Germany). A venous blood sample was taken at 16:00 hours to determine the serum C-peptide level.

Statistical analyses Data are shown as mean $\pm \mathrm{SD}$ or median with range, level of significance was $p=0.05$. SPSS 13 (SPSS Inc., Chicago, IL, USA) was used for all statistical analyses. A two-factor repeated measures ANOVA with Greenhouse-Geisser correction was performed to determine the differences in glucose levels between the three insulin preparations. Glucose level comparisons at single time points were performed by paired Student's $t$ tests. As these tests are primarily descriptive, no corrections for multiple comparisons were performed. Comparisons of duration of hypoglycaemia and serum Cpeptide levels were done using the Friedman test. 


\section{Results}

Patients Mean $\mathrm{HbA}_{1 \mathrm{c}}$ was $7.3 \pm 0.7 \%$ at the time of recruitment. At the time of the fasting test, mean $\mathrm{HbA}_{1 \mathrm{c}}$ was $7.2 \pm 0.6 \%$ for $\mathrm{NPH}$ insulin, $6.8 \pm 0.6 \%$ for insulin detemir and $6.8 \pm 0.9 \%$ for insulin glargine. The mean weight of the patients remained constant during the study. At the time of recruitment, $17(85 \%)$ of the patients were treated with NPH insulin and three $(15 \%)$ were treated with insulin detemir; none had been treated with insulin glargine. The mean dose of the long-acting insulin at the time of the fasting test was $29 \pm 10 \mathrm{U}$ for NPH insulin, $33 \pm 13 \mathrm{U}$ for insulin detemir and $32 \pm 12 \mathrm{U}$ for insulin glargine.

Glucose concentrations from 24:00 to 07:00 hours Glucose profiles (determined by CGMS) are shown in Fig. 1. The mean glucose levels achieved at 24:00 and 07:00 hours were comparable for all three insulin preparations. The mean glucose concentration at 24:00 hours was 5.8 $1.7 \mathrm{mmol} / 1$ for $\mathrm{NPH}$ insulin, $6.0 \pm 1.7 \mathrm{mmol} / 1$ for insulin detemir and $6.1 \pm 2.1 \mathrm{mmol} / 1$ for insulin glargine. At 07:00 hours the glucose concentrations between the groups were also similar: $6.7 \pm 1.7 \mathrm{mmol} / 1$ for $\mathrm{NPH}$ insulin, $6.7 \pm$ $1.0 \mathrm{mmol} / 1$ for insulin detemir and $6.9 \pm 1.8 \mathrm{mmol} / \mathrm{l}$ for insulin glargine. There were no statistically significant $(p=$ 0.125 ) differences in mean glucose values between the various insulin preparations from 24:00 to 07:00 hours.

Symptomatic nocturnal hypoglycaemia occurred twice, once with NPH insulin and once with insulin glargine. These tests were repeated on another day with a reduced

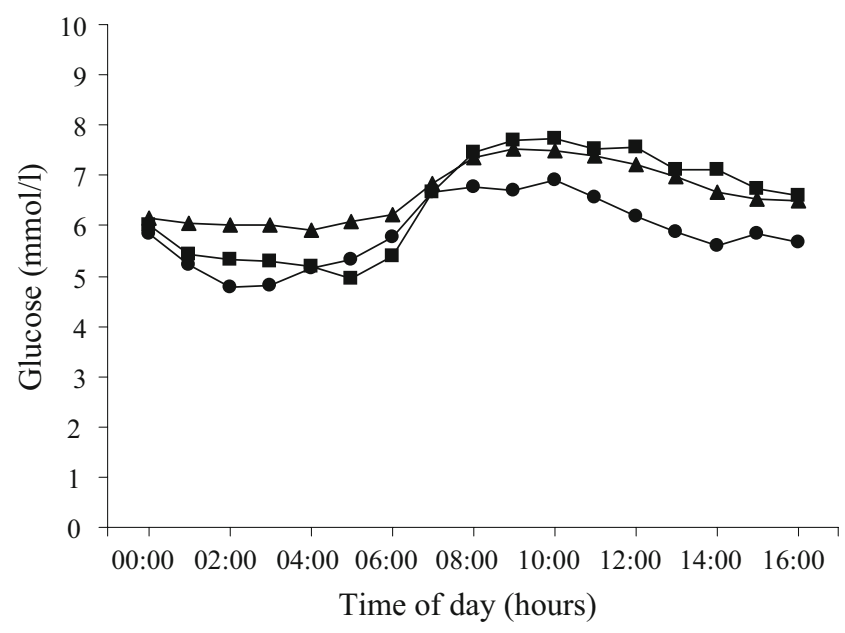

Fig. 1 Mean glucose concentrations measured by CGMS at hourly intervals in 20 patients with type 2 diabetes during the fasting test. The long-acting insulins (NPH insulin, circles; insulin detemir, squares; insulin glargine, triangles) were injected at 22:45 hours; breakfast and lunch were not consumed the following day. Bars indicating SDs (in the range of $20-35 \%$ of the mean values) have been removed because they were overlapping to such an extent that they were not discernible for the three individual curves dose of insulin. The median duration of glucose values of $<3 \mathrm{mmol} / \mathrm{l}$ (according to CGMS) in asymptomatic patients was 130 (35-230) $\mathrm{min}$ for NPH insulin (four patients), 235 (200-270) min for insulin glargine (two patients) and 30 (10-30) $\mathrm{min}$ for insulin detemir (three patients). These differences were not statistically significant.

Glucose concentrations from 08:00 to 16:00 hours Glucose concentrations (determined by CGMS) increased between 05:00 and 07:00 hours with all insulin preparations tested (Fig. 1). The mean glucose concentrations measured between 08:00 and 16:00 hours differed significantly ( $p=$ 0.032 ) between the different insulin preparations; they were lower for NPH than for insulin detemir $(p=0.012)$ and insulin glargine $(p=0.049)$. At 16:00 hours the glucose concentrations in those treated with NPH insulin tended to be lower $(5.7 \pm 1.5 \mathrm{mmol} / \mathrm{l}, p=0.08)$ than in those treated with insulin detemir $(6.6 \pm 1.4 \mathrm{mmol} / \mathrm{l})$ or insulin glargine $(6.5 \pm 1.9 \mathrm{mmol} / \mathrm{l})$. Neither symptomatic hypoglycaemia nor a glucose level of $<3 \mathrm{mmol} / 1$ measured by CGMS occurred in any patient during the day. Median serum C-peptide levels at 16:00 hours were lower for NPH insulin (200 [20$610] \mathrm{pmol} / \mathrm{l}, p=0.006)$ than for insulin detemir (285 [180$640] \mathrm{pmol} / \mathrm{l})$ or insulin glargine (320 [50-750] pmol/1).

\section{Discussion}

Predefined target glucose levels (at 24:00 and 07:00 hours) could be reached with all three tested long-acting insulin preparations, with a trend for lower dose requirements for $\mathrm{NPH}$ compared with insulin analogues. The absolute differences in glucose concentrations (by CGMS) overnight and the next morning in response to the three different insulin preparations were minor and of questionable clinical relevance; the frequency of low nocturnal glucose levels did not differ widely, and no hypoglycaemia occurred during the next day when meals were omitted. Hence, all three long-acting insulin preparations seem to be appropriate for maintaining glucose levels within a desirable range overnight in patients with type 2 diabetes, although none could totally prevent glucose levels from rising between 05:00 and 08:00 hours.

Our small study had essentially no power to detect potential differences between the three long-acting insulin preparations with regard to longer term glycaemic control $\left(\mathrm{HbA}_{1 \mathrm{c}}\right)$ and changes in body weight. The glucose concentrations measured at night were consistent with the known profiles of the different insulin preparations. The length of time glucose concentrations were $<3 \mathrm{mmol} / \mathrm{l}$ was lowest with insulin detemir, and the glucose nadir was observed earlier (at 02:00 hours) after NPH insulin than after insulin 
detemir (05:00 hours) or insulin glargine (04:00 hours) injection.

Despite the theoretically shorter duration of action of $\mathrm{NPH}$ insulin compared with insulin detemir and insulin glargine $[1-3,10]$, glucose levels following bedtime injection were lower during the next day following NPH insulin than after either of the insulin analogues. The longer biological action of NPH insulin was also supported by the lower serum C-peptide concentrations observed at 16:00 hours after injection of NPH insulin compared with injection of either of the insulin analogues. This finding may seem counterintuitive and surprising at first sight, but it shows that study results described in patients with type 1 diabetes cannot be extrapolated to patients with type 2 diabetes who are overweight and insulin-resistant and exhibit significant residual insulin secretion.

The major limitation of our study is that treatment order was not randomised. $\mathrm{HbA}_{1 \mathrm{c}}$ decreased slightly during the study and NPH insulin was tested first in most patients, i.e. when $\mathrm{HbA}_{1 \mathrm{c}}$ tended to be higher. A trend for lower NPH dose requirements (especially in obese patients on high total doses) to reach glucose targets was observed. It remains unclear whether our findings can be explained by the sequence of insulin testing.

Another limitation of our study is that glucose concentrations were determined by CGMS. However, available plasma glucose concentrations from capillary blood samples confirmed the CGMS data (data not shown).

The data suggest that (1) the risk of hypoglycaemia after bedtime injection of NPH insulin, insulin detemir or insulin glargine when breakfast and lunch are omitted the next day is low; (2) NPH insulin is not inferior to insulin detemir or insulin glargine when used in an appropriate manner for the treatment of patients with type 2 diabetes.

Acknowledgements We thank H. Seiler for technical assistance.
Duality of interest This study was financially supported by an unrestricted grant from Abbott, Sanofi-Aventis, Novo-Nordisk and Roche Diagnostics.

\section{References}

1. Heise T, Nosek L, Rønn B et al (2004) Lower within-subject variability of insulin detemir in comparison to NPH insulin and insulin glargine in people with type 1 diabetes. Diabetes 53:16141620

2. Plank J, Bodenlenz M, Sinner F et al (2005) A double-blind, randomized, dose-response study investigating the pharmacodynamic and pharmacokinetic properties of the long-acting insulin analog detemir. Diabetes Care 28:1107-1112

3. Lepore M, Pampanelli S, Fanelli C et al (2000) Pharmacokinetics and pharmacodynamics of subcutaneous injection of long-acting human insulin analog glargine, NPH insulin, and ultralente human insulin and continuous subcutaneous infusion of insulin lispro. Diabetes 49:2142-2148

4. Swinnen S, Holleman F, DeVries J (2008) The interpretation of glucose clamp studies of long-acting insulin analogues: from physiology to marketing and back. Diabetologia 51:1790-1795

5. Holleman F, Gale E (2007) Nice insulins, pity about the evidence. Diabetologia 50:1783-1790

6. Trümper B, Reschke K, Molling J (1995) Circadian variation of insulin requirement in insulin dependent diabetes mellitus the relationship between circadian change in insulin demand and diurnal patterns of growth hormone, cortisol and glucagon during euglycemia. Horm Metab Res 27:141-147

7. Shapiro E, Polonsky K, Copinschi G et al (1991) Nocturnal elevation of glucose levels during fasting in noninsulin-dependent diabetes. J Clin Endocrinol Metab 72:444-454

8. Wiesli P, Lehmann R, Krayenbuehl P, Schmid C, Spinas G (2006) Assessment of basal insulin requirement using fasting tests in insulin-treated patients with type 2 diabetes mellitus. Exp Clin Endocrinol Diabetes 114:539-543

9. Cockcroft D, Gault M (1976) Prediction of creatinine clearance from serum creatinine. Nephron 16:31-41

10. Heinemann L, Linkeschova R, Rave K, Hompesch B, Sedlak M, Heise T (2000) Time-action profile of the long-acting insulin analog insulin glargine (HOE901) in comparison with those of NPH insulin and placebo. Diabetes Care 23:644-649 